\title{
Histological Observations on the Formation of Meristematic Colonies Derived from Lettuce (Lactuca sativa L.) Protoplasts
}

\author{
Ritsuko Murakamı* and Seibi OKA**
}

Nishio et $a l .{ }^{1)}$, using an osmoticum of $0.3 \mathrm{M}$ sucrose and gelrite-beads method, noticed that very compact colonies with a smooth surface structure were formed in modified Murashige and Skoog ${ }^{2)}$ medium supplemented with $1 \mathrm{mg} / l \quad \alpha$-naphthaleneacetic acid(NAA) and $0.3 \mathrm{mg} / l$ 6-benzyladenine (BA) from cotyledon-derived protoplasts of lettuce. When the colonies having this meristematic structure (hereafter referred to as MC) were selected and transferred to shoot inducing medium, they produced shoot buds at a high frequency. We also confirmed the formation of MC. Further interest was that the structure emerged at a very early stage of colony growth. There have been only a limited number of histological studies on colony growth at the initial stage of protoplast culture $^{5)}$. In this paper we histologically compared the formation of $\mathrm{MC}$ and non-meristematic colony (NMC) under two different hormonal conditions.

Lettuce seeds (cv. Kaiser) were aseptically sown on MS medium containing $2 \%$ sucrose and 0.2 $\%$ gelrite. They were germinated at $23 \pm 1.5^{\circ} \mathrm{C}$ under a $16 \mathrm{hr}$ light photoperiod (3, $000 \mathrm{lux}$ ). Fiveday-old cotyledons were treated with an enzyme solution containing $0.5 \%$ Cellulase Onozuka RS, $0.025 \%$ Pectolyase Y23 and $0.5 \mathrm{M}$ mannitol ( $\mathrm{pH} \mathrm{5.8)}$ ) at $25-30^{\circ} \mathrm{C}$ for $16 \mathrm{hr}$ in darkness. Protoplast isolation and culture were performed basically according to the method of Nishio et al. ${ }^{11}$. The protoplasts were cultured under the dark at $25-30^{\circ} \mathrm{C}$. The $\mathrm{pH}$ of the medium was adjusted to 6.0 before autoclaving at $120^{\circ} \mathrm{C}$ for $15 \mathrm{~min}$. The protoplasts were embedded in medium containing $0.3 \%$ gelrite at a final density of $1 \times 10^{4} / \mathrm{m} l$ unless otherwise mentioned. The gelrite blocks were transferred to $15 \mathrm{~m} l$ of liquid media immediately after the soldification of the medium. The liquid medium had the same components as the solid medium. After 7 days, the liquid medium was replaced by a fresh liquid medium with a reduced concentration of sucrose (0.15 M). Colony size, plating efficiency, and the frequency of MC formation were visually assessed after 2 weeks of culture. The developmental processes of colony growth were compared between $\mathrm{MC}$ forming $(1$ $\mathrm{mg} / l \mathrm{NAA}+1 \mathrm{mg} / l \mathrm{BA})$ and $\mathrm{NMC}$ forming $(0.1 \mathrm{mg} / l \mathrm{NAA}+1 \mathrm{mg} / l \mathrm{BA})$ medium. Small pieces of gelrite block $(10 \times 10 \times 1 \mathrm{~mm})$ sampled every 2 days for 2 weeks were fixed in FAA (90: 5: 5/70\% ethanol: acetic acid: formalin), dehydrated by $t$-butanol series, and embedded in Paraplast plus. Serial sections $(10 \mu \mathrm{m})$ were stained with safranin and first green ${ }^{3)}$. At least three specimens were subjected to microscopic examination for each sampling time.

The procedures of protoplast culture by Nishio et al. ${ }^{1)}$ were slightly improved in terms of the hormonal condition and transfer of gelrite blocks to liquid medium. BA concentration was elevated from $0.3 \mathrm{mg} / l$ to $1 \mathrm{mg} / l$, because $\mathrm{MC}$ was most frequently formed in the medium supplemented with $1 \mathrm{mg} / l \mathrm{NAA}+1 \mathrm{mg} / \mathrm{l} \mathrm{BA}$. By contrast most of the colonies proved to become NMC when the concentration of NAA was reduced to $0.1 \mathrm{mg} / l$. Nishio $e$ t al. ${ }^{1}{ }^{1}$ transferred the gelrite blocks to liquid medium 7-10 days after culture. This method, however, still induced some secre-

* National Institute of Sericultural and Entomological Science, Ohwashi 1-2, Tsukuba, Ibaraki 305, Japan

** National Institute of Agrobiological Resources, Kannondai 2-1-2, Tsukuba, Ibaraki 305, Japan 


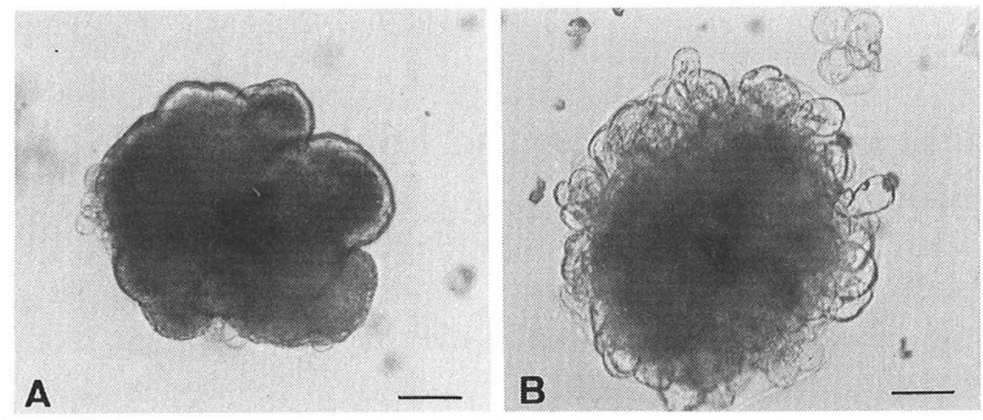

Fig. 1 Meristematic colony (MC) and non-meristematic colony (NMC) derived from lettuce protoplasts after 10 days of culture.

A, MC formed in medium containing $1.0 \mathrm{mg} / l \mathrm{NAA}$ and $1.0 \mathrm{mg} / l \mathrm{BA} . \mathrm{B}, \mathrm{NMC}$ formed in medium containing $0.1 \mathrm{mg} / l \mathrm{NAA}$ and $1.0 \mathrm{mg} / l \mathrm{BA}$. (Scale bar $=100 \mu \mathrm{m}$ )
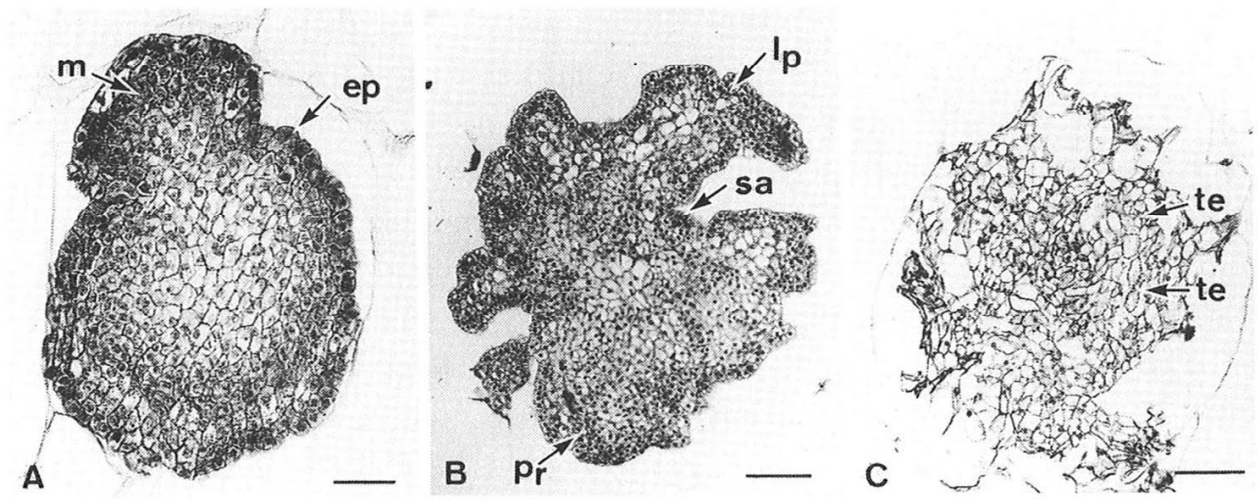

Fig. 2 Histology of MC and NMC development.

A, MC after 10 days of culture. B, MC after 14 days of culture. C, NMC after 14 days of culture. m: meristematic cells, ep: epidermis, lp: leaf primordium, te: tracheary element, pr: protuberance. sa: shoot apex. Scale bar $=50 \mu \mathrm{m}(\mathrm{A})$ and $100 \mu \mathrm{m}(\mathrm{B}, \mathrm{C})$.

tion of yellow substance(s) into the medium that eventually retarded subsequent colony growth, particularly when protoplasts were cultured at cell densities higher than $2.5 \times 10^{4} / \mathrm{m} l$. This problem was overcome by transferring the gelrite blocks into liquid medium immediately after solidifying the protoplast medium-gelrite mixture. This procedure enabled over $50 \%$ of the colonies to develop into $\mathrm{MC}$ even at a relatively high cell density of $2.5 \times 10^{4} / \mathrm{m} l$.

Protoplast density remarkably affected the frequency of $\mathrm{MC}$ formation as well as hormonal factors. Higher plating efficiency and higher frequency of $\mathrm{MC}$ formation were obtained at a lower cell density. Although the highest frequency of $\mathrm{MC}$ formation was observed at $1 \times 10^{4} / \mathrm{m} l$, protoplasts were cultured at $2.5 \times 10^{4} / \mathrm{m} l$ for histological study, because this afforded a greater number of $\mathrm{MC}$ in a given volume of specimen, resulting in its easier microscopic detection. Reduced plating efficiencies at higher protoplast densities in lettuce protoplast culture were also obtained in previous studies $^{1,4)}$.

MC having unique compact and smooth-surfaced structures appeared after 6-10 days of culture and were easily discernible from NMC which featured loosely combined cells in the periphery of the colony (Fig. 1-A, B). When histologically examined, the morphological differences between the two types of colonies were evident as early as 4-6 days after culture. MC was composed of smaller size cells with a more prominent nucleus than NMC. After 10 days of culture, MC developed to 
differentiate a distinct epidermal structure and meristematic cells in several parts of the subepidermal region of the colony (Fig. 2-A). After 12-14 days of culture, the round shape of MC changed in ways associated with the formation of protuberances, some of which further developed into shoot apices including leaf primordia from 14 days onwards after culture(Fig. 2-B). During the culture period, NMC only increased in size and were composed of enlarged cells with a number of well differentiated tracheary elements(TE), but without any epidermal structures (Fig. 2-C). These TE were scattered at random in the center of the colony without forming organized vascular systems and were usually accompanied by air space. Although less frequently than in NMC, TE was also formed after 8 days of culture in MC, adjacent to the meristematic cells toward the center of the colony. The finding that shoot formation occurred at a high frequency when selected MC were transferred to shoot differentiation medium ${ }^{1)}$ was histologically confirmed by the successive formation of leaf primordia from the protuberances produced in MC.

In this investigation, we could not find localized initial cells leading to $\mathrm{MC}$ in a colony at any particular time of its growth; hence the actively dividing cells which produced small cells seemed, as a whole, responsible for the formation of $\mathrm{MC}$ from the very beginning of the protoplast culture. In conclusion, the present study revealed that lettuce protoplasts are characterized by the direct differentiation of meristematic structures in $\mathrm{MC}$ at quite an early stage (6-8 days) of culture without prominent, intervenient callus formation, and subsequent early differentiation of shoot buds (from 14 days onwards).

(Accepted May 21, 1996)

\section{References}

1) Nishio, T., T. Sato, K. Mori, K. Takayanagi, 1988. Japan. J. Breed., 38: 165-171.

2) Murashige, T., F. Skoog, 1962. Physiol. Plant., 15: 473-497.

3) Yeung, E. C., R. L. Peterson, 1972. Can. J. Bot., 50: 73-78.

4) Engler, D. E., R. G. Grogan, 1982/83. Plant Science Letters, 28: 223-229.

5) Christiane, F., G. Hahne, 1992. Protoplasma, 169: 130-138. 